\title{
THE POSITRON EXCESS AND SUPERSYMMETRIC DARK MATTER*
}

\author{
EDWARD A. BALTZ ${ }^{\dagger}$ \\ ISCAP, Columbia Astrophysics Laboratory, $550 \mathrm{~W}$ 120th St., Mail Code 5247, \\ New York, NY 10027, USA \\ $\underline{\text { JOAKIM EDSJÖ) }}^{\ddagger}$ \\ Department of Physics, Stockholm University, AlbaNova, SE-106 91 Stockholm, \\ Sweden \\ KATHERINE FREESE ${ }^{\S}$ \\ Michigan Center for Theoretical Physics, Physics Department, University of \\ Michigan, Ann Arbor, MI 48109, USA \\ PAOLO GONDOLO \\ Dept. of Physics, Case Western Reserve University, 10900 Euclid Ave., \\ Cleveland, $\mathrm{OH}$ 44106-7079, USA
}

Using a new instrument, the HEAT collaboration has confirmed the excess of cosmic ray positrons that they first detected in 1994. We explore the possibility that this excess is due to the annihilation of neutralino dark matter in the galactic halo. We confirm that neutralino annihilation can produce enough positrons to make up the measured excess only if there is an additional enhancement to the signal. We quantify the 'boost factor' that is required in the signal for various models in the Minimal Supersymmetric Standard Model parameter space, and find that a boost factor $\geq 30$ provides good fits to the HEAT data. Such an enhancement in the signal could arise if we live in a clumpy halo.

\footnotetext{
*Preprint: CWRU-P15-02

†E-mail: eabaltz@physics.columbia.edu

‡Rapporteur, E-mail: edsjo@physto.se

$\S$ E-mail: ktfreese@umich.edu

IE-mail: pxg26@po.cwru.edu
} 


\section{Introduction}

Several years ago the HEAT collaboration reported an excess of cosmic ray positrons with energies $\sim 10 \mathrm{GeV}^{1}$. In 2000 they again measured this excess using a new instrument, and found excellent agreement ${ }^{2}$. The possibility that this excess is due to annihilations of Weakly Interacting Massive Particles (WIMPs) in the galactic halo, in particular the neutralinos in supersymmetric models, has been investigated by many authors; the positron fluxes have been calculated previously ${ }^{3,4,5,6,7,8}$, and attempts to fit the new HEAT data with positrons from supersymmetric dark matter annihilations have been made ${ }^{9,10,11}$.

In an earlier work ${ }^{7}$ two of us calculated the positron fluxes from neutralino annihilation in the halo and compared with the excess reported then by HEAT ${ }^{1}$. Recently we investigated the possibility that the HEAT excess (in light of the new data) could be due to neutralinos ${ }^{9}$. We will here go through this possibility and comment on some other work along the same lines.

\section{Supersymmetric model}

We work in the Minimal Supersymmetric Standard Model (MSSM), with the usual low-energy parameters: the Higgsino mass parameter $\mu$, the gaugino mass parameter $M_{2}$, the ratio of the Higgs vacuum expectation values $\tan \beta$, the mass of the $C P$-odd Higgs boson $m_{A}$, the scalar mass parameter $m_{0}$ and the trilinear soft SUSY-breaking parameters $A_{b}$ and $A_{t}$ for the third generation. More details are presented elsewhere ${ }^{12,13}$.

We will consider the case when the lightest neutralino is the lightest supersymmetric particle and we will use DarkSUSY ${ }^{14}$ for our calculations. We calculate the relic density including coannihilations between neutralinos and charginos ${ }^{13,15}$. We will here only include models where $0.05 \leq \Omega_{\chi} h^{2} \leq$ $0.25\left(\Omega_{\chi}\right.$ is the density in units of the critical density and $h$ is the present Hubble constant in units of $100 \mathrm{~km} \mathrm{~s}^{-1} \mathrm{Mpc}^{-1}$ ).

We have used the scans over the MSSM parameter space developed in our earlier work ${ }^{9}$. For each model, we have check if it is excluded by any accelerator constraints. The most important of these are the LEP bounds ${ }^{17}$ on the lightest chargino mass $\left(88.4 \mathrm{GeV}\right.$ if $\left|m_{\chi_{1}^{+}}-m_{\chi_{1}^{0}}\right|>3$ and $67.7 \mathrm{GeV}$ otherwise) and on the lightest Higgs boson mass $m_{h}$ (which ranges from 91.5-112 GeV depending on $\tan \beta$ ) and the constraints from $b \rightarrow s \gamma^{18}$ (we use the LO implementation in DarkSUSY ${ }^{14}$ ). 


\section{Positron spectra and fits to the HEAT data}

We obtain the positron flux from neutralino annihilation in the galactic halo $^{7}$. The model is a true diffusion model and assumes that the diffusion region of tangled galactic magnetic field is an infinite slab. This approximation is reasonable since most of the positrons are emitted quite nearby so that the outer radial boundary is unimportant. Furthermore, energy losses due to synchrotron radiation and inverse Compton scattering from the cosmic microwave background and from starlight are included. This model roughly agrees with earlier work ${ }^{4}$, though the inclusion of inverse Compton scattering from starlight is crucial as it doubles the energy loss rate.

In calculating the observed positron flux from annihilations in the halo, we encounter several astrophysical uncertainties. First, cosmic ray propagation is not perfectly understood, though the errors are unlikely to be larger than a factor of two. More importantly, the structure of the galactic dark halo is unknown. Any clumpiness in the halo serves to enhance the signal. There is no compelling argument for any particular value of the enhancement factor, be it unity or in the thousands or more.

We will parameterize the clumpiness as a boost factor, $B_{s}$, which we define to be the boost factor that the WIMP annihilation signal from a smooth galactic halo must be multiplied by to match the HEAT data. However, we must be careful that in postulating a boost factor, we do not overproduce the other products of neutralino annihilation, especially antiprotons and gamma rays ${ }^{19}$. We do have some freedom here, in that the boost factors for positrons, antiprotons and gamma rays are not necessarily equal, as their propagation is not the same. It turns out that for a distribution of clumps that follow that of the smooth component, the antiproton flux is not boosted as much at the positron flux ${ }^{19,9}$.

We fit the full positron dataset of the HEAT experiments (1994 and 1995 combined data ${ }^{1}$ and the 2000 data $^{2}$ ).

We will in the following assume that the standard prediction for the positron background ${ }^{20,21}$ is correct to within a normalization factor $N$. We find that the best fit normalization of the background with no signal from neutralinos is $N=1.14$, with $\chi^{2}=3.33$ per degree of freedom. When adding the signal, we make a simultaneous fit of the normalization of the background $N$ and the normalization of the signal $B_{s}$, for each supersymmetric model in our database of models. We say that a given model "gives a good fit to the positron data" when: (1) the background-plus-signal fit 

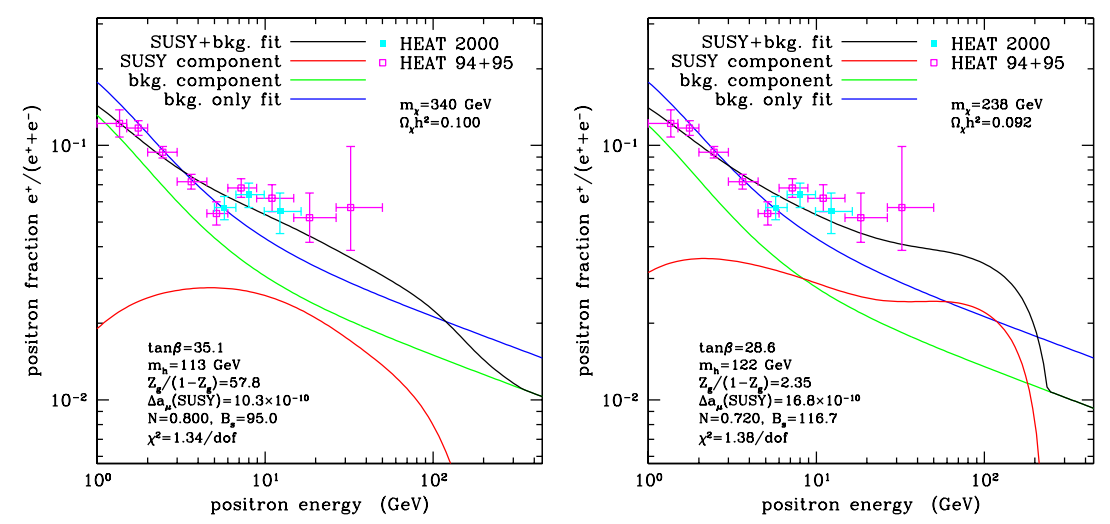

Figure 1. Positron fraction data and fits. We illustrate positron data from HEAT 94+95 and HEAT 2000, a background only fit, and a SUSY+background fit for two interesting MSSM models. Two additional curves separately display the SUSY and background components of the combined SUSY+background fit. These models are gaugino dominated and the model in a) has positrons primarily from hadronization, while the model in b) has hard positrons from direct gauge boson decays.

fits the data better than the background-only fit with a decrease in $\chi^{2}$ per degree of freedom greater than unity, namely the background-plus-signal fit has $\chi^{2} \leq 2.33$ per degree of freedom; (2) the best fit normalization of the background $N$ is between 0.5 and 2.0, namely the background calculation ${ }^{20}$ is correct to within a factor of two according to the best fit.

The positron fluxes are more than an order of magnitude smaller than the HEAT measurements, and we find that the best fit normalizations of the signal $B_{s}$ lie between 30 and $10^{10}$. Values of $B_{s}$ as large as $10^{10}$ are hardly realistic, but $B_{s}$ up to 100-1000 might be acceptable given the uncertainties in the halo structure. For each model we than check that, with this boost factor $B_{s}$, it is not excluded by other experiments. As mentioned earlier, the antiprotons are the most restrictive in this sense and we have applied the antiproton constraint saying that the standard calculation of the antiproton flux (boosted with an antiproton boost factor derived from $B_{s}{ }^{9}$ ) does not yield more than at most a factor of 5 too high antiproton fluxes compared to the BESS measurements ${ }^{22}$. The factor of 5 is chosen as an upper limit on the propagation uncertainties.

In Fig. 1 we plot the positron data from the HEAT 94+95 and HEAT 2000 experiments, together with the background only fit, and two interesting SUSY models that have good fits. The apparent sharp increase in the 
positron fraction around $7 \mathrm{GeV}$ is not evident in any of our SUSY models, even before the smoothing effects of energy loss on the spectrum. In principle one could envision a sharp edge coming from the direct decay of gauge bosons. However, we would then get positrons also from the hadronic decay of the gauge bosons and this would entirely dominate the flux at $7 \mathrm{GeV}$ as seen in Fig. 1b.

Kane et al. show that even a monochromatic signal of positrons would not be able to reproduce the sharp bump seen in the data ${ }^{10}$. A monochromatic signal is smeared by energy losses and does not give the sharp feature indicated in the data. However, a way to sharpen the bump would be to have them all come from a nearby clump which is smaller than the propagation length. Then a line signal would not be smeared out. This problem has not yet been treated in depth, but is a bit less appealing since it requires fine-tuning of both the particle physics and the astrophysics model to explain the data.

\section{Conclusions}

We have explored models in the Minimal Supersymmetric Standard Model (MSSM) parameter space to find how large the boost factor must be for each of the models. The lowest boost factor we found is roughly 30 for a WIMP that is primarily a Bino in content with mass $160 \mathrm{GeV}$. For $B_{s}<100$, we find that the models are gaugino-dominated, though some have significant Higgsino fractions. The masses of the models are in the range $150-400 \mathrm{GeV}$ for the most part. For $100<B_{s}<1000$, the masses are as large as $2 \mathrm{TeV}$, and some very pure Higgsinos become allowed.

Fitting the new HEAT data with positrons from supersymmetric dark matter has been done by other groups ${ }^{10,11}$ where they come to more or less the same conclusions, i.e. that large boost factors are needed and that the fits are better than the background only fits, but they are not excellent.

\section{Acknowledgments}

J.E. thanks the Swedish Research Council for support.

\section{References}

1. S. W. Barwick et al. (HEAT Collaboration), Astrophys. J. 482 (1997) L191.

2. S. Coutu et al. (HEAT-pbar Collaboration), in Proceedings of 27th ICRC (2001). 
3. J. Silk and M. Srednicki, Phys. Rev. Lett. 53 (1984) 624; S. Rudaz and F. Stecker, Astrophys. J. 325 (1988) 16; J. Ellis, R.A. Flores, K. Freese, S. Ritz, D. Seckel and J. Silk, Phys. Lett. B214 (1989) 403; F. Stecker and A. Tylka, Astrophys. J. 336 (1989) L51; D. Eichler Phys. Rev. Lett. 63 (1989) 2440.

4. M. Kamionkowski and M. S. Turner, Phys. Rev. D43 (1991) 1774.

5. M.S. Turner and F. Wilczek, Phys. Rev. D42 (1990) 1001; A.J. Tylka, Phys. Rev. Lett. 63 (1989) 840.

6. S. W. Barwick et al. (HEAT Collaboration), Astrophys. J. 498 (1998) 779; S. Coutu et al. (HEAT Collaboration), Astropart. Phys. 11 (1999) 429.

7. E. A. Baltz and J. Edsjö, Phys. Rev. D59 (1999) 023511.

8. I. V. Moskalenko and A. W. Strong, Phys. Rev. D60 (1999) 063003.

9. E.A. Baltz, J. Edsjö, K. Freese and P. Gondolo, Phys. Rev. D65 (2001) 063511.

10. G.L. Kane, L.-T. Wang and J. D. Wells, Phys. Rev. D65 (2002) 057701; G.L. Kane, L.-T. Wang and T.T. Wang, Phys. Lett. B536 (2002) 263.

11. W. de Boer, C. Sander, M. Horn and D. Kazakov, astro-ph/0207557.

12. L. Bergström and P. Gondolo, Astropart. Phys. 5 (1996) 263.

13. J. Edsjö and P. Gondolo, Phys. Rev. D56 (1997) 1879.

14. P. Gondolo, J. Edsjö, L. Bergström, P. Ullio, and E.A. Baltz, astro$\mathrm{ph} / 0012234$.

15. P. Gondolo and G. Gelmini, Nucl. Phys. B360 (1991) 145.

16. X. Wang, M. Tegmark and M. Zaldarriaga, Phys. Rev. D65 (2002) 123001.

17. D. E. Groom et al. (Particle Data Group), Eur. Phys. J. C, 15, 1 (2000).

18. M. S. Alam et al. (CLeo Collaboration), Phys. Rev. Lett. 71 (1993) 674 and Phys. Rev. Lett. 74 (1995) 2885.

19. L. Bergström, J. Edsjö, P. Gondolo, and P. Ullio, Phys. Rev. D59 (1999) 043506.

20. I. V. Moskalenko and A. W. Strong, Astrophys. J. 493 (1998) 694.

21. I. V. Moskalenko, A. W. Strong, J. F. Ormes and M. S. Potgieter, Astrophys. J. 565 (2002) 280.

22. S. Orito et al., Phys. Rev. Lett. 84 (2000) 1078. 